\title{
A Metric for Quantitative Analysis of Vascular Tortuosity
}

\author{
Glen DeLoid*, Niraj Trivedi**, Jean Lai*, Renee Dickie*, and Rick A. Rogers*,** \\ *BioMedical Imaging Laboratory, Department of Environmental Health, Harvard School of Public \\ Health, 665 Huntington Ave, Boston, MA \\ ** Vascular Biology Program, Department of Surgical Research, Children's Hospital Boston, Karp \\ Family Research Building, Boston, MA 02215
}

Blood vessels within cancerous tissues are known to exhibit increased tortuosity[1-4]. A reliable technique for measuring vascular tortuosity could therefore be valuable in the identification of diseased tissue boundaries. A number of methods have been proposed for the quantification of blood vessel tortuosity from microscopic image data, but no single metric robustly defines all possible vessel geometries. The Distance Metric (DM), which is the ratio of vessel path length to distance between endpoints, is highly sensitive to amplitude, but relatively insensitive to wavelength, such that a gradual high amplitude curve can produce equal or greater values than a clearly more tortuous short wavelength sinusoidal with a smaller amplitude[1]. The Inflection Count Metric (ICM), which is the distance metric multiplied by the number of inflection points, discriminates between sinusoids of different wavelengths, but does not distinguish between coils since they contain no inflection points. This is a significant drawback since coiled microvessels are commonly observed in newly formed vessels or in vessels undergoing angiogenesis[5]. The Sum of Angles Metric (SOAM), the mean of combined intersegment and torsional angles measured at equal intervals along the vessel path, does not correctly differentiate between high and low amplitude coils or sinusoids[1]. We note two important limitations of the SOAM metric. First, the torsional angle component of the sum is problematic, since its value increases greatly (approaching pi) in the vicinity of inflections, and is unnecessary, since the intersegment angle is itself a three-dimensional angle. Second, the measurement of these angles at equal intervals along the vessel path with subsequent division by the pathlength serves to negate the contribution of pathlength (a strong indicator of tortuosity).

We have developed a metric that combines elements of the SOAM and DM metrics and that appears to measure tortuosity consistently for sinusoidal and coiled geometries. Because intersegment angles adequately represent the 3-dimensional bending of a vessel, torsional angles were eliminated from the calculation. To address the issue of division by pathlength, we measured angles at equally-spaced intervals along a line between the vessel endpoints rather than along the vessel path. The resulting mean intersegment angle was multiplied by the distance metric to add greater amplitude sensitivity.

The combined Product of Angle and Distance Metric, PADM, was tested with phantom sinusoidal and coil vessels. Figure 1 shows that for both sinusoidal and coil shapes the PADM metric exhibited a balanced sensitivity to wavelength and amplitude. For example, a sinusoid with an amplitude of 20 microns and wavelength of 100 microns had an PADM value roughly equal to that of a sinusoid with amplitude 10 microns and wavelength 75 microns (the lower amplitude was offset by the shorter wavelength). 
Tortuosity analysis of blood vessels from organ fat pads, brain, heart and skeletal muscle using the PADM appropriately defined the vascular bed in these tissues and supported the PADM as a valid utility. Vessels ranging from nearly straight to highly tortuous were blindly scored by experienced vascular researchers and evaluated by the PADM method (figure 2). These results demonstrate a close correspondence between apparent tortuosity and the PADM calculation.

\section{References}

[1] E. Bullitt et al., Medical Image Analysis. 9 (2005) 39.

[2] E. Bullitt et al., IEEE-Trans. Med. Imag. 22 (2003) 1163.

[3] J. Folkman, J. Natl. Cancer Inst. 92 (2000) 94.

[4] J.S. Baish and R. K. Jain, Cancer Research. 60 (2000) 3683.

[5] B. Dome et al., J Neuropathol Exp Neurol. 62(6) (2003) 655

[6] This research was supported by NIH PO1 CA 45548, NIH HL 65960, and NIH ES 00002.

A

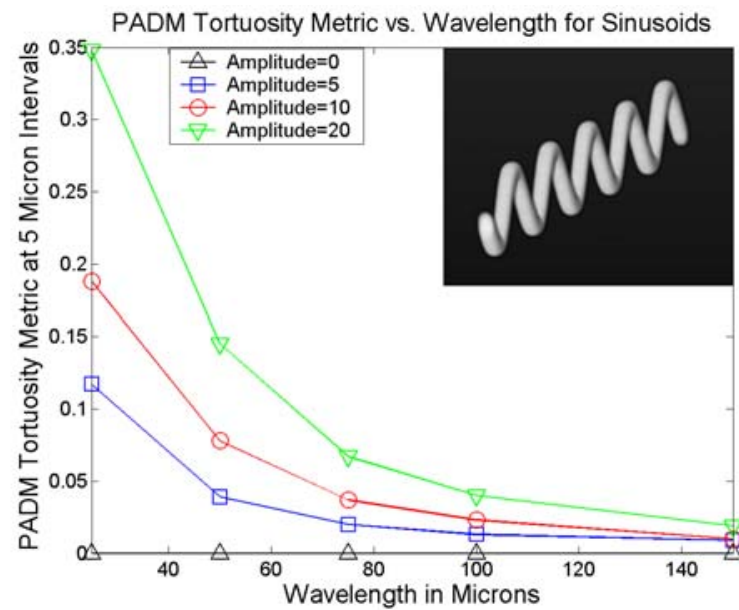

B

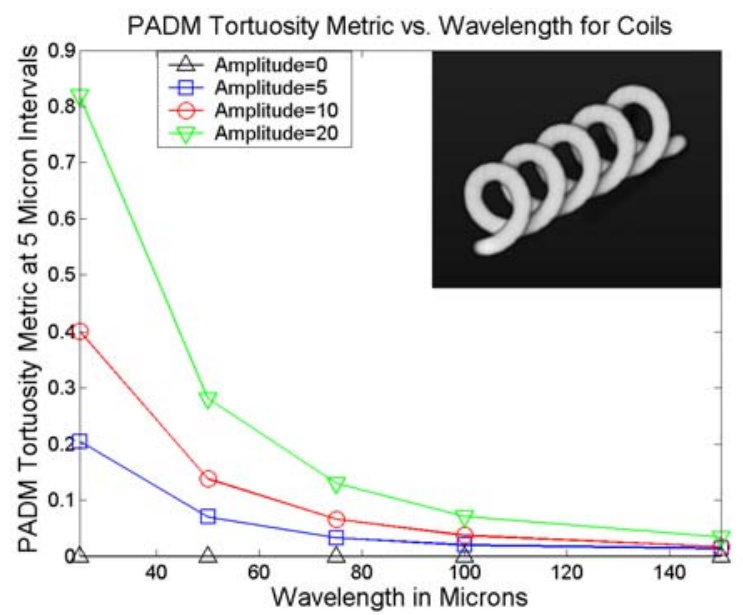

Fig. 1. PADM results for phantom data. A. sinusoids. B. coils.

A

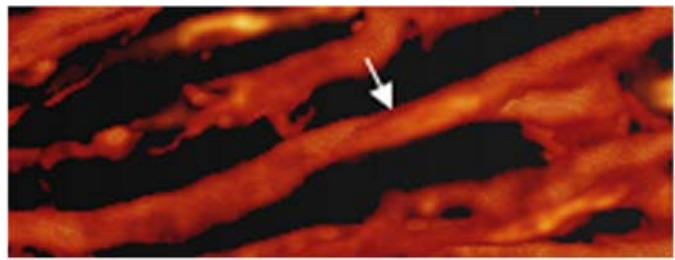

B

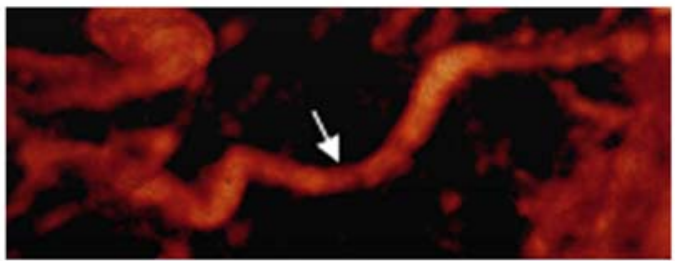

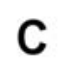

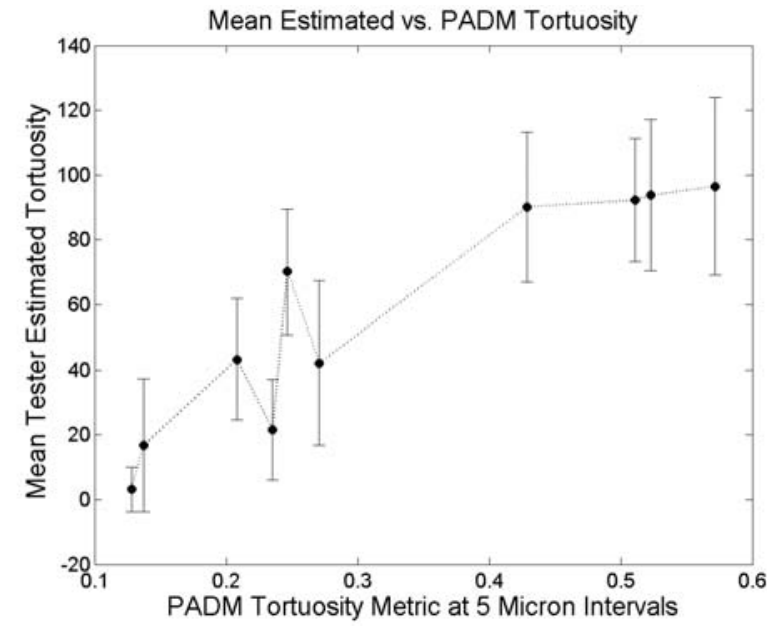

Fig. 2. A and B. 3D reconstructions of vessels from microscopic sections. A. mouse myocardium. B. cerebral glial tumor. C. Comparison of PADM and scored tortuosity for vessels from myocardium, skeletal muscle, brain, fat pad and cerebral glial tumor. Error bars represent $+/$ - one standard deviation. 